\title{
p-ADIC PROPERTIES OF MAASS FORMS ARISING FROM THETA SERIES
}

\author{
Sharon Anne Garthwaite and David Penniston
}

\begin{abstract}
We investigate arithmetic properties of the Fourier coefficients of certain harmonic weak Maass forms of weight $1 / 2$ and $3 / 2$. Each of the forms in question is the sum of a holomorphic function and a period integral of a theta series. In particular, for any positive integer $M$ coprime to 6 we prove that the coefficients of the holomorphic function satisfy Ramanujan-type congruences modulo $M$, and establish sufficient conditions under which they are well-distributed modulo $\ell^{j}$ for primes $\ell \geq 5$. As an example we show that our results apply to Ramanujan's mock theta function $\omega(q)$.
\end{abstract}

\section{Introduction}

The theory of modular forms has been instrumental in the study of many arithmetic functions. For example, let $p(n)$ denote the partition function, that is, the number of ways to express $n$ as the sum of a nonincreasing sequence of positive integers. S. Ahlgren and K. Ono $[1,4,16]$ used modular forms to prove that for any positive integer $M$ with $(M, 6)=1$, there are infinitely many arithmetic progressions $A n+B$, none of which is a subprogression of another, such that

$$
p(A n+B) \equiv 0 \quad(\bmod M)
$$

for all $n \geq 0$. In addition to divisibility, one naturally wonders about the distribution of $p(n)$ modulo $M$. In the 1960s M. Newman conjectured that for any positive integer $M$ and any integer $0 \leq r<M, p(n) \equiv r(\bmod M)$ for infinitely many values of $n$. In $2003 \mathrm{~J}$. Bruinier and Ono [12] proved the validity of Newman's conjecture for any prime $M \geq 13$ assuming the truth of Ramanujan's conjectured nonexistence of a congruence of the form $p(M n+B) \equiv 0(\bmod M)$, and Ahlgren and M. Boylan [2] completed the proof by showing that such congruences exist only when $M \in\{5,7,11\}$.

The results mentioned above depend on general theorems regarding the Fourier coefficients of integral and half-integral weight holomorphic modular forms. It is possible to extend such results to larger classes of functions. For example, S. Treneer $[19,20]$ has shown that congruences exist for the Fourier coefficients of a large class of weakly holomorphic modular forms. In this paper we consider the parallel theory for a class of harmonic weak Maass forms (see Definition 2.1). In recent papers, K. Bringmann, A. Folsom, Ono and R. Rhoades [8, 9, 11] have constructed families of such forms of weight $1 / 2$ and $3 / 2$ related to theta series of weight $3 / 2$ and $1 / 2$, respectively. (See [15] for a survey of their results.) In addition, Bringmann and Ono

Received by the editors April 18, 2007.

2000 Mathematics Subject Classification. 11F30, 11F37.

The first author was partially supported by the University of Wisconsin-Madison NSF VIGRE program. 
$[8,9]$ show how to construct weakly holomorphic modular forms from Maass forms in these families, and use their construction to obtain congruences for Dyson's rank, a simple partition statistic. (For further rank congruences see [6].) Here we extend these results to a general class of harmonic weak Maass forms which arise from theta series in the sense of Definition 2.2. The forms we consider have Fourier expansions of the shape

$$
f(z)=\sum_{n=n_{0}}^{\infty} a(n) q^{n}+\sum_{n=0}^{\infty} \beta_{n}(y) q^{-d n^{2}}
$$

where $q:=e^{2 \pi i z}$ with $z:=x+i y(x, y \in \mathbb{R}), d$ is a positive integer, $a(n) \in \mathbb{C}$ and the functions $\beta_{n}: \mathbb{R}^{+} \rightarrow \mathbb{C}$ are as defined in Section 3. As a consequence we obtain the following description of congruences satisfied by the Fourier coefficients $a(n)$. For $p$ an odd prime denote by $\chi_{p}$ the Legendre symbol modulo $p$, i.e., $\chi_{p}(\bullet):=\left(\frac{\bullet}{p}\right)$.

Theorem 1.1. Suppose $k \in\left\{\frac{1}{2}, \frac{3}{2}\right\}, N$ is a positive integer divisible by 4 and $f(z)$ is a weight $k$ harmonic weak Maass form on $\Gamma_{1}(N)$. Suppose further that $f(z)$ is good as in Definition 2.2, with Fourier expansion (1.1). Let $p$ be any odd prime with $p \nmid d$. Then for any prime $\ell \nmid 3 N p$ and any $j \geq 1$, there exists an integer $m \geq 1$ such that $a$ positive proportion of the primes $Q$ have the property that

$$
a\left(Q^{3} \ell^{m} n\right) \equiv 0 \quad\left(\bmod \ell^{j}\right)
$$

for all $n$ with $(n, \ell Q)=1$ and $\chi_{p}\left(-Q^{3} \ell^{m} d n\right)=-1$.

Moreover, when $f(z)$ is a Maass form on $\Gamma_{0}(N)$ with real Nebentypus, we obtain the following theorem regarding the distribution of coefficients in nonzero residue classes.

Theorem 1.2. Suppose $f(z)$ and $p$ satisfy the hypotheses of Theorem 1.1. Suppose further that $f(z)$ is a harmonic weak Maass form on $\Gamma_{0}(N)$ with real Nebentypus $\chi$, $K=\mathbb{Q}, \ell \nmid 3 N p$ is prime and $j \geq 1$. Then there exists an integer $m \geq 1$ such that if we define the coefficients $b(n)$ modulo $\ell^{j}$ by

$$
\sum_{n=1}^{\infty} b(n) q^{n}:=\sum_{\ell \nmid n, \chi_{p}\left(-\ell^{m} d n\right)=-1} a\left(\ell^{m} n\right) q^{n}
$$

and $\sum_{n=1}^{\infty} b(n) q^{n} \not \equiv 0(\bmod \ell)$, then at least one of the following is true.

(1) If $0 \leq r<\ell^{j}$, then

$$
\#\left\{0 \leq n \leq X: a(n) \equiv r \quad\left(\bmod \ell^{j}\right)\right\} \gg_{r, \ell, j} \begin{cases}\sqrt{X} / \log (X) & \text { if } r \neq 0 \\ X & \text { if } r=0\end{cases}
$$

(2) There is a finite set of squarefree integers $\left\{n_{1}, n_{2}, \ldots, n_{t}\right\}$ such that

$$
\sum_{n=1}^{\infty} b(n) q^{n} \equiv \sum_{i=1}^{t} \sum_{m=1}^{\infty} b\left(n_{i} m^{2}\right) q^{n_{i} m^{2}} \quad(\bmod \ell)
$$


Theorem 1.2 extends analogous results for half-integral weight modular forms. (See Theorem 1 of [3], Theorem 1 of [12] and Theorem 1 of [13]).

As an application of Theorems 1.1 and 1.2 we consider the mock theta functions defined by Ramanujan [18]. Examples include the "third order" functions

$$
\begin{aligned}
f(q):=\sum_{n=0}^{\infty} \alpha_{f}(n) q^{n}: & :=1+\sum_{n=1}^{\infty} \frac{q^{n^{2}}}{(1+q)^{2}\left(1+q^{2}\right)^{2} \cdots\left(1+q^{n}\right)^{2}} \\
& =1+q-2 q^{2}+3 q^{3}-3 q^{4}+3 q^{5}-5 q^{6}+\cdots
\end{aligned}
$$

and

$$
\begin{aligned}
\omega(q):=\sum_{n=0}^{\infty} \alpha_{\omega}(n) q^{n}: & =\sum_{n=0}^{\infty} \frac{q^{2 n^{2}+2 n}}{(1-q)^{2}\left(1-q^{3}\right)^{2} \cdots\left(1-q^{2 n+1}\right)^{2}} \\
& =1+2 q+3 q^{2}+4 q^{3}+6 q^{4}+8 q^{5}+10 q^{6}+\cdots .
\end{aligned}
$$

In 2002-2003, S. P. Zwegers [21, 22] proved that the mock theta functions can be "completed" to real analytic weight $1 / 2$ vector-valued modular forms, and Bringmann, Ono and Rhoades $[9,10,11]$ have related mock theta functions to weight $1 / 2$ harmonic weak Maass forms. In Corollary 4.2 we prove that $2 q^{2} \omega\left(q^{3}\right)$ bears a similar relation to a weight $1 / 2$ harmonic weak Maass form. Theorem 1.1 then allows us to conclude that for every positive integer $M$ with $(M, 6)=1$, there are infinitely many arithmetic progressions $A n+B$, none of which is contained in another, so that

$$
\alpha_{\omega}(A n+B) \equiv 0 \quad(\bmod M)
$$

for all $n \geq 0$. (note. Though not explicitly stated, the work of Bringmann and Ono implies such results for $\alpha_{f}(n)$.) Similarly, Theorem 1.2 gives sufficient conditions for $\alpha_{\omega}(n)$ to be well-distributed modulo powers of primes $\ell \geq 5$.

The proofs of the main theorems involve extending standard techniques from the theory of modular forms. In Section 2 we set notation and state definitions. In Section 3 we prove our results by establishing a connection between harmonic weak Maass forms arising from theta series and holomorphic modular forms. In Section 4 we apply this theory to $\alpha_{\omega}(n)$.

\section{Preliminaries}

Here we recall relevant background on harmonic weak Maass forms. We begin by introducing notation. Throughout this paper we assume $k \in \frac{1}{2}+\mathbb{Z}$. Given $A=$ $\left(\begin{array}{ll}a & b \\ c & d\end{array}\right) \in G L_{2}^{+}(\mathbb{Q})$, let $A$ act on the complex upper half-plane $\mathbb{H}$ in the usual way, that is,

$$
A z:=\frac{a z+b}{c z+d}
$$

for $z \in \mathbb{H}$. Write $z=x+i y$ with $x \in \mathbb{R}, y \in \mathbb{R}^{+}$, and let

$$
\Delta_{k}:=-y^{2}\left(\frac{\partial^{2}}{\partial x^{2}}+\frac{\partial^{2}}{\partial y^{2}}\right)+i k y\left(\frac{\partial}{\partial x}+i \frac{\partial}{\partial y}\right)
$$


be the weight $k$ hyperbolic Laplacian. Let $\Gamma$ be a subgroup of $\Gamma_{0}(4)$ of finite index, and define $\epsilon_{d}$ for $d$ odd by

$$
\epsilon_{d}:=\left\{\begin{array}{lll}
1 & \text { if } d \equiv 1 & (\bmod 4) \\
i & \text { if } d \equiv 3 & (\bmod 4)
\end{array}\right.
$$

Definition 2.1. We call a smooth function $f: \mathbb{H} \rightarrow \mathbb{C}$ a harmonic weak Maass form of weight $k$ on $\Gamma$ if it satisfies the following three conditions.

(1) $f(A z)=\left(\frac{c}{d}\right)^{2 k} \epsilon_{d}^{-2 k}(c z+d)^{k} f(z)$ for all $A \in \Gamma$.

(2) $\Delta_{k} f=0$.

(3) $f$ has at most linear exponential growth at each cusp of $\Gamma$.

Denote by $\operatorname{Maass}_{k}(\Gamma)$ the space of harmonic weak Maass forms of weight $k$ on $\Gamma$, and by $\operatorname{Weak}_{k}(\Gamma)$ the subspace consisting of weight $k$ weakly holomorphic modular forms on $\Gamma$. (Recall that weakly holomorphic modular forms are meromorphic modular forms whose poles, if any, are supported on the cusps.) Note that if $f \in \operatorname{Maass}_{k}(\Gamma)$, then $f \in \operatorname{Weak}_{k}(\Gamma)$ if and only if $f$ is holomorphic on $\mathbb{H}$.

Let $N$ be a positive integer divisible by 4 and let $\chi$ be a Dirichlet character modulo $N$. We denote by $\operatorname{Maass}_{k}\left(\Gamma_{0}(N), \chi\right)$ the space of forms $f \in \operatorname{Maass}_{k}\left(\Gamma_{1}(N)\right) \operatorname{satisfying}$

$$
f(A z)=\chi(d)\left(\frac{c}{d}\right)^{2 k} \epsilon_{d}^{-2 k}(c z+d)^{k} f(z)
$$

for all $A \in \Gamma_{0}(N)$, and refer to $\chi$ as the Nebentypus of $f$. Denote by $\operatorname{Weak}_{k}\left(\Gamma_{0}(N), \chi\right)$ the subspace of forms in $\operatorname{Maass}_{k}\left(\Gamma_{0}(N), \chi\right)$ which are holomorphic on $\mathbb{H}$.

For $j \in \frac{1}{2} \mathbb{Z}$ and $\Gamma^{\prime}$ a congruence subgroup of $\mathrm{SL}_{2}(\mathbb{Z})$ of level $M$ (with $4 \mid M$ if $j \notin \mathbb{Z}$ ) we denote by $M_{j}\left(\Gamma^{\prime}\right)$ the space of holomorphic modular forms on $\Gamma^{\prime}$, and by $S_{j}\left(\Gamma^{\prime}\right)$ the subspace of $M_{j}\left(\Gamma^{\prime}\right)$ consisting of cusp forms. Finally, for a Dirichlet character $\chi$ modulo $M$ we denote by $M_{j}\left(\Gamma_{0}(M), \chi\right)\left(\operatorname{resp} . S_{j}\left(\Gamma_{0}(M), \chi\right)\right)$ the space of holomorphic modular (resp. cusp) forms on $\Gamma_{0}(M)$ with Nebentypus $\chi$.

Now suppose $k \in\left\{\frac{1}{2}, \frac{3}{2}\right\}$. For integers $a, b$ with $b>0$ and a Dirichlet character $\chi$ with conductor $b$, let

$$
g_{\chi}:=\sum_{n=0}^{b-1} \chi(n) e^{2 \pi i n / b}
$$

be the Gauss sum with respect to $\chi$, and let

$$
\theta_{k, a, b}(z):=\sum_{n \equiv a} n_{(\bmod b)}^{k-\frac{1}{2}} q^{n^{2}}
$$

and

$$
\Theta_{k, \chi}(z):=\sum_{n \in \mathbb{Z}} \chi(n) n^{k-\frac{1}{2}} q^{n^{2}}
$$

be the usual weight $k$ theta series, where $\chi$ is even (resp. odd) if $k=\frac{1}{2}$ (resp. $k=\frac{3}{2}$ ). Finally, denote by $\xi_{k}$ the differential operator

$$
\xi_{k}:=y^{k} \frac{\bar{\partial}}{\partial \bar{z}} .
$$


Definition 2.2. Suppose $f(z) \in \operatorname{Maass}_{k}(\Gamma)$. We say that $f(z)$ is good if there exist positive integers $b, d$ and complex numbers $\rho_{a, b}(0 \leq a<b)$ such that the following two conditions are satisfied.

(1) $\xi_{k}(f(z))=\sum_{a=0}^{b-1} \rho_{a, b} \theta_{2-k, a, b}(d z)$.

(2) $a(n) \in \mathcal{O}_{K}$ in the Fourier expansion (1.1) of $f(z)$ for some number field $K$.

Remark. In the proof of Proposition 3.1 we show that condition (1) in Definition 2.2 implies that $f(z)$ has a Fourier expansion of the form (1.1).

Remark. In [9, 11] Bringmann, Ono and Rhoades construct families of good harmonic weak Maass forms of weight 1/2. In [8] Bringmann, Folsom and Ono show that for any $a, b$ with $b \geq 4$ even and $a$ coprime to $b$ it is possible to construct a good harmonic weak Maass form $f_{a, b}(z)$ of weight $3 / 2$ such that $\xi_{3 / 2}\left(f_{a, b}(z)\right)$ is a constant multiple of $\theta_{\frac{1}{2}, a, b}(z)$, and in [7] Bringmann, Bruinier, Folsom and Ono prove a similar result for $\theta_{\frac{3}{2}, a, b}(z)$.

\section{Proofs of main results}

In this section we prove Theorems 1.1 and 1.2. We begin with a preliminary proposition. Recall that $\chi_{p}(\bullet)=\left(\frac{\bullet}{p}\right)$ for odd primes $p$.

Proposition 3.1. Let $k \in\left\{\frac{1}{2}, \frac{3}{2}\right\}$, and let $N$ be a positive integer divisible by 4 . Suppose $f(z) \in \operatorname{Maass}_{k}\left(\Gamma_{1}(N)\right)$ is good and has Fourier expansion (1.1). Then for every odd prime $p$ with $p \nmid d$,

$$
w_{p}(z):=\sum_{\chi_{p}(-n d)=-1} a(n) q^{n} \in \operatorname{Weak}_{k}\left(\Gamma_{1}\left(N p^{4}\right)\right) .
$$

Moreover, if $f(z) \in \operatorname{Maass}_{k}\left(\Gamma_{0}(N), \chi\right)$, then $w_{p}(z) \in \operatorname{Weak}_{k}\left(\Gamma_{0}\left(N p^{4}\right), \chi\right)$.

Proof. We begin by studying the Fourier expansion of $f(z)$. By Definition 2.2, there exist coefficients $c(n) \in \mathbb{C}$ such that

$$
\xi_{k}(f(z))=\sum_{n=0}^{\infty} c(n) q^{d n^{2}} .
$$

Observing that

$$
\xi_{k}\left(\int_{-\bar{z}}^{i \infty} \frac{e^{2 \pi i n \tau}}{(-i(\tau+z))^{k}} d \tau\right)=y^{k} \cdot \frac{\overline{e^{-2 \pi i n \bar{z}}}}{(-i(-\bar{z}+z))^{k}}=2^{-k} e^{2 \pi i n z},
$$

we find that

It follows that

$$
\xi_{k}\left(\int_{-\bar{z}}^{i \infty} \frac{\sum_{n=0}^{\infty} 2^{k} \overline{c(n)} e^{2 \pi i d n^{2} \tau}}{(-i(\tau+z))^{k}} d \tau\right)=\xi_{k}(f(z)) .
$$

$$
f(z)-\int_{-\bar{z}}^{i \infty} \frac{\sum_{n=0}^{\infty} 2^{k} \overline{c(n)} e^{2 \pi i d n^{2} \tau}}{(-i(\tau+z))^{k}} d \tau
$$

is holomorphic on $\mathbb{H}$. Now, under the change of variables $u=-i(\tau+z)$,

$$
\int_{-\bar{z}}^{i \infty} \frac{e^{2 \pi i d n^{2} \tau}}{(-i(\tau+z))^{k}} d \tau=i e^{-2 \pi i d n^{2} z} \int_{2 y}^{\infty} \frac{e^{-2 \pi d n^{2} u}}{u^{k}} d u .
$$


Recalling the definition of the incomplete gamma function

$$
\Gamma(a, x):=\int_{x}^{\infty} e^{-t} t^{a-1} d t
$$

we have that $f(z)$ has a Fourier expansion of the form (1.1), where

$$
\beta_{n}(y):=2^{2 k-1} i \pi^{k-1} d^{k-1} \overline{c(n)} n^{2 k-2} \Gamma\left(1-k, 4 \pi d n^{2} y\right) .
$$

In particular, the non-holomorphic part of $f(z)$ is supported on powers of $q$ that are $-d$ times squares.

In analogy with the case of modular forms, for an odd prime $p$ and a function $h: \mathbb{H} \rightarrow \mathbb{C}$ define the $p$ th quadratic twist of $h$ by

$$
\left(h \otimes \chi_{p}\right)(z):=\frac{g_{\chi_{p}}}{p} \sum_{v=0}^{p-1} \chi_{p}(v) h\left(\gamma_{v} z\right)
$$

where $\gamma_{v}:=\left(\begin{array}{cc}1 & -v / p \\ 0 & 1\end{array}\right)$. Note that if $h$ has a Fourier expansion of the form

$$
h(z)=\sum_{n \in \mathbb{Z}} \delta_{n}(y) q^{n}
$$

for functions $\delta_{n}: \mathbb{R}^{+} \rightarrow \mathbb{C}$, then

$$
\begin{aligned}
\left(h \otimes \chi_{p}\right)(z) & =\frac{g_{\chi_{p}}}{p} \sum_{v=0}^{p-1} \chi_{p}(v) \sum_{n \in \mathbb{Z}} \delta_{n}(y) e^{2 \pi i n(z-v / p)} \\
& =\frac{g_{\chi_{p}}}{p} \sum_{n \in \mathbb{Z}} \delta_{n}(y)\left(\sum_{v=0}^{p-1} \chi_{p}(v) e^{-2 \pi i n v / p}\right) q^{n} .
\end{aligned}
$$

Since

$$
\sum_{v=0}^{p-1} \chi_{p}(v) e^{-2 \pi i n v / p}=\chi_{p}(-n) \sum_{v=0}^{p-1} \chi_{p}(-n v) e^{-2 \pi i n v / p}=\chi_{p}(-n) g_{\chi_{p}}
$$

and $g_{\chi_{p}}^{2}=\chi_{p}(-1) p$, it follows that

$$
\left(h \otimes \chi_{p}\right)(z)=\sum_{n \in \mathbb{Z}} \chi_{p}(n) \delta_{n}(y) q^{n} .
$$

As each summand in the Fourier expansion (1.1) has the form (3.1), we find that

$$
\left(f \otimes \chi_{p}\right)(z)=\sum_{n \geq n_{0}} \chi_{p}(n) a(n) q^{n}+\chi_{p}(-d) \sum_{p \nmid n} \beta_{n}(y) q^{-d n^{2}} .
$$

Just as in the case of modular forms, one can show that if $h \in \operatorname{Maass}_{k}\left(\Gamma_{1}(M)\right)$ (resp. $\left.\operatorname{Maass}_{k}\left(\Gamma_{0}(M), \psi\right)\right)$, then $h \otimes \chi_{p} \in \operatorname{Maass}_{k}\left(\Gamma_{1}\left(M p^{2}\right)\right)\left(\operatorname{resp} \operatorname{Maass}_{k}\left(\Gamma_{0}\left(M p^{2}\right), \psi\right)\right)$.

Since $p \nmid d$, we see from (3.2) that the form

$$
f(z)-\chi_{p}(-d)\left(f \otimes \chi_{p}\right)(z) \in \operatorname{Maass}_{k}\left(\Gamma_{1}\left(N p^{2}\right)\right)
$$

has Fourier expansion

$$
\sum_{p \mid n} a(n) q^{n}+\sum_{\chi_{p}(-d n)=-1} 2 a(n) q^{n}+\sum_{p \mid n} \beta_{n}(y) q^{-d n^{2}}
$$


and therefore

$$
w_{p}(z):=-\frac{\chi_{p}(-d)}{2}\left(\left(f-\chi_{p}(-d)\left(f \otimes \chi_{p}\right)\right) \otimes \chi_{p}\right)(z) \in \operatorname{Maass}_{k}\left(\Gamma_{1}\left(N p^{4}\right)\right)
$$

has Fourier expansion

$$
\sum_{\chi_{p}(-d n)=-1} a(n) q^{n}
$$

Since this is a holomorphic function on $\mathbb{H}$, it follows that $w_{p}(z) \in \operatorname{Weak}_{k}\left(\Gamma_{1}\left(N p^{4}\right)\right)$, with $w_{p}(z) \in \operatorname{Weak}_{k}\left(\Gamma_{0}\left(N p^{4}\right), \chi\right)$ if $f(z) \in \operatorname{Maass}_{k}\left(\Gamma_{0}(N), \chi\right)$.

The main results of our paper are implied by the following theorem.

Theorem 3.2. Suppose $f(z)$ and $p$ are as in Proposition 3.1, and suppose also that $j \geq 1, \ell$ is prime and $\ell \nmid 3 N p$. Then there exist nonnegative integers $\lambda$ and $m$ and $a$ half-integer weight cusp form

$$
w_{p, \ell, m, j}(z) \in S_{\lambda+\frac{1}{2}}\left(\Gamma_{1}\left(144 N p^{4} \ell^{2}\right)\right)
$$

such that

$$
w_{p, \ell, m, j}(z) \equiv \sum_{\ell \nmid n, \chi_{p}\left(-\ell^{m} d n\right)=-1} a\left(\ell^{m} n\right) q^{n} \quad\left(\bmod \ell^{j}\right) .
$$

Further, if $f(z) \in \operatorname{Maass}_{k}\left(\Gamma_{0}(N), \chi\right)$, then $w_{p, \ell, m, j}(z) \in S_{\lambda+\frac{1}{2}}\left(\Gamma_{0}\left(144 N p^{4} \ell^{2}\right), \chi \chi_{\ell}^{m}\right)$.

Proof. Let $w_{p}(z)$ be the weakly holomorphic form whose existence is guaranteed by Proposition 3.1. If $f(z) \in \operatorname{Maass}_{k}\left(\Gamma_{0}(N), \chi\right)$, then the result follows by applying Theorem 3.1 of [20] to $w_{p}(z)$.

For the general situation we utilize the theory of newforms. Denote by $\eta$ Dedekind's eta function

$$
\eta(z):=q^{1 / 24} \prod_{n=1}^{\infty}\left(1-q^{n}\right)
$$

Since $\eta(24 z) \in S_{1 / 2}\left(\Gamma_{0}(576), \chi_{12}\right)$ (where $\left.\chi_{12}(\bullet)=(\underline{12})\right)$ is a cusp form and the poles of $w_{p}(z)$ are supported at the cusps, we can find a nonnegative integer $v$ such that

$$
\eta^{2 v+1}(24 z) w_{p}(z) \in S_{k+v+\frac{1}{2}}\left(\Gamma_{1}\left(144 N p^{4}\right)\right) .
$$

Note that since the Fourier coefficients of $\eta^{2 v+1}(24 z)$ are in $\mathbb{Z}$, the Fourier coefficients of $\eta^{2 v+1}(24 z) w_{p}(z)$ are in $\mathcal{O}_{K}$, and as the latter is an integer weight cusp form, there exist algebraic integers $\alpha_{i}$ and positive integers $\delta_{i}$ such that

$$
\eta^{2 v+1}(24 z) w_{p}(z)=\sum_{i=1}^{r} \alpha_{i} u_{i}\left(\delta_{i} z\right)
$$

where $u_{i}(z)$ is a newform of level $L_{i}$ dividing $144 N p^{4}$ and $\delta_{i} \mid\left(144 N p^{4} / L_{i}\right)$. Write

$$
t_{i}(z):=\alpha_{i} u_{i}\left(\delta_{i} z\right) / \eta^{2 v+1}(24 z)
$$

and denote by $L$ be the number field obtained by adjoining $\alpha_{i}$ and the Fourier coefficients of $u_{i}(z)$ to $K$ for all $1 \leq i \leq r$. Then we have $w_{p}(z)=\sum_{i=1}^{r} t_{i}(z)$ written as a sum of forms in $\operatorname{Weak}_{k}\left(\Gamma_{1}\left(144 N p^{4}\right)\right.$ ), each with Nebentypus (say $t_{i}(z)$ has Nebentypus $\psi_{i}$ ) and with Fourier coefficients lying in $\mathcal{O}_{L}$. 
Fix $1 \leq i \leq r$, and for positive integers $t$ and functions $h: \mathbb{H} \rightarrow \mathbb{C}$ define the usual linear operators $U_{t}$ and $V_{t}$ by

$$
\left(h \mid U_{t}\right)(z):=\frac{1}{t} \sum_{v=0}^{t-1} h\left(\frac{z+v}{t}\right)
$$

and

$$
\left(h \mid V_{t}\right)(z):=h(t z) .
$$

Write $t_{i}(z)=\sum a_{i}(n) q^{n}$. By Proposition 3.5 of [20] there is an integer $m_{i}$ such that for $m \geq m_{i}$ the function

$$
t_{i}\left|U_{\ell^{m}}(z)-t_{i}\right| U_{\ell^{m+1}} \mid V_{\ell}(z)=\sum_{\ell \nmid n} a_{i}\left(\ell^{m} n\right) q^{n}
$$

lies in $\operatorname{Weak}_{k}\left(\Gamma_{0}\left(144 N p^{4} \ell^{2}\right), \psi_{i} \chi_{\ell}^{m}\right)$ and vanishes at every cusp $\frac{a}{c}$ of $\Gamma_{0}\left(144 N p^{4} \ell^{2}\right)$ with $\ell^{2} \mid c$.

Now fix $m \geq \max \left\{m_{i}: 1 \leq i \leq r\right\}$. The form

$$
F_{\ell}(z):=\frac{\eta^{\ell^{2}}(z)}{\eta\left(\ell^{2} z\right)} \in M_{\frac{\ell^{2}-1}{2}}\left(\Gamma_{0}\left(\ell^{2}\right)\right)
$$

vanishes at every cusp $\frac{a}{c}$ of $\Gamma_{0}\left(144 N p^{4} \ell^{2}\right)$ with $\ell^{2} \nmid c$ (see [17], p. 18) and has the property that $F_{\ell}^{\ell^{s-1}}(z) \equiv 1\left(\bmod \ell^{s}\right)$ for any $s \geq 1$. It follows that if we choose $s \geq j-1$ sufficiently large, then for any $1 \leq i \leq r$,

$$
\left(t_{i}\left|U_{\ell^{m}}(z)-t_{i}\right| U_{\ell^{m+1}} \mid V_{\ell}(z)\right) \cdot F_{\ell}^{\ell^{s-1}}(z)
$$

is a form in $S_{\lambda+\frac{1}{2}}\left(\Gamma_{0}\left(144 N p^{4} \ell^{2}\right), \psi_{i} \chi_{\ell}^{m}\right)$, where $\lambda:=k+\frac{\ell^{s-1}\left(\ell^{2}-1\right)-1}{2} \in \mathbb{Z}$. Fix such an $s$ and define

$$
w_{p, \ell, m, j}(z):=\left(w_{p}\left|U_{\ell^{m}}(z)-w_{p}\right| U_{\ell^{m+1}} \mid V_{\ell}(z)\right) \cdot F_{\ell}^{\ell^{s-1}}(z) .
$$

It follows by linearity that $w_{p, \ell, m, j}(z) \in S_{\lambda+\frac{1}{2}}\left(\Gamma_{1}\left(144 N p^{4} \ell^{2}\right)\right)$ and

$$
w_{p, \ell, m, j}(z) \equiv \sum_{\ell \nmid n, \chi_{p}\left(-\ell^{m} d n\right)=-1} a\left(\ell^{m} n\right) q^{n} \quad\left(\bmod \ell^{j}\right) .
$$

We now prove Theorems 1.1 and 1.2.

Proof of Theorem 1.1. Let $w_{p, \ell, m, j}(z)$ be the cusp form whose existence is guaranteed by Theorem 3.2. By Lemma 3.1 of [4], a positive proportion of the primes $Q \equiv-1$ $\left(\bmod 144 N p^{4} \ell^{j+2}\right)$ have the property that

$$
w_{p, \ell, m, j}(z) \mid T\left(Q^{2}\right) \equiv 0 \quad\left(\bmod \ell^{j}\right),
$$

where $T\left(Q^{2}\right)$ is the usual Hecke operator on $S_{\lambda+\frac{1}{2}}\left(\Gamma_{1}\left(144 N p^{4} \ell^{2}\right)\right)$. For ease of notation write $w_{p, \ell, m, j}(z)=\sum b(n) q^{n}$. The Fourier expansion of $w_{p, \ell, m, j}(z) \mid T\left(Q^{2}\right)$ is then

$$
\sum\left(b\left(Q^{2} n\right)+Q^{\lambda-1}\left(\frac{(-1)^{\lambda} n}{Q}\right) b(n)+Q^{2 \lambda-1} b\left(n / Q^{2}\right)\right) q^{n} .
$$


If $Q$ exactly divides $n$, then the coefficient of $q^{n}$ in this expansion is $b\left(Q^{2} n\right)$. Therefore $a\left(Q^{3} \ell^{m} n\right) \equiv 0\left(\bmod \ell^{j}\right)$ provided that

$$
(n, \ell Q)=1 \quad \text { and } \quad \chi_{p}\left(-Q^{3} \ell^{m} d n\right)=-1 .
$$

Proof of Theorem 1.2. Since $f$ is a harmonic weak Maass form on $\Gamma_{0}(N)$ with Nebentypus $\chi$, Theorem 3.2 implies the existence of a form

$$
w_{p, \ell, m, j}(z) \in S_{\lambda+\frac{1}{2}}\left(\Gamma_{0}\left(144 N p^{4} \ell^{2}\right), \chi \chi_{\ell}^{m}\right)
$$

with

$$
w_{p, \ell, m, j}(z) \equiv \sum_{\ell \nmid n, \chi_{p}\left(-\ell^{m} d n\right)=-1} a\left(\ell^{m} n\right) q^{n} \quad\left(\bmod \ell^{j}\right) .
$$

The result follows by applying Theorem 1 of [3] to $w_{p, \ell, m, j}(z)$.

Remark. Our proof of Theorem 3.2 employs work of Treneer and the theory of newforms to associate a cusp form to the weight $k$ weakly holomorphic modular form $w_{p}(z)$ furnished by Proposition 3.1. This argument applies to any half-integer weight weakly holomorphic form on $\Gamma_{1}(N)$ with coefficients in $\mathcal{O}_{K}$, and thus results like Theorems 1.1 and 1.2 hold for such a form.

\section{The coefficients of $\omega(q)$}

As an example of the consequences of Theorem 1.1 we consider Ramanujan's third order mock theta function $\omega(q)$ defined by (1.3). Our goal is to prove the existence of congruences for $\alpha_{\omega}(n)$ similar to those satisfied by the partition function. We will do this by showing that $2 q^{2} \omega\left(q^{3}\right)$ is related to a good weight $1 / 2$ harmonic weak Maass form.

We begin by recalling a result of Zwegers. Let $f(q)$ be the mock theta function defined by (1.2). Throughout this section let $e(x):=e^{2 \pi i x}$. For $z \in \mathbb{H}$ define a vector-valued function $F(z)$ by

$$
F(z):=\left(F_{0}(z), F_{1}(z), F_{2}(z)\right)^{T}:=\left(q^{-\frac{1}{24}} f(q), 2 q^{\frac{1}{3}} \omega\left(q^{\frac{1}{2}}\right), 2 q^{\frac{1}{3}} \omega\left(-q^{\frac{1}{2}}\right)\right)^{T} .
$$

Similarly, for $z \in \mathbb{H}$ define a vector-valued non-holomorphic function $G(z)$ by

$$
G(z):=\left(G_{0}(z), G_{1}(z), G_{2}(z)\right)^{T}:=2 i \sqrt{3} \int_{-\bar{z}}^{i \infty} \frac{\left(g_{1}(\tau), g_{0}(\tau),-g_{2}(\tau)\right)^{T}}{\sqrt{-i(\tau+z)}} d \tau
$$

where the $g_{i}(\tau)$ are the cuspidal weight $3 / 2$ theta functions

$$
\begin{aligned}
& g_{0}(\tau):=\sum_{n=-\infty}^{\infty}(-1)^{n}\left(n+\frac{1}{3}\right) e^{3 \pi i\left(n+\frac{1}{3}\right)^{2} \tau}, \\
& g_{1}(\tau):=-\sum_{n=-\infty}^{\infty}\left(n+\frac{1}{6}\right) e^{3 \pi i\left(n+\frac{1}{6}\right)^{2} \tau}, \\
& g_{2}(\tau):=\sum_{n=-\infty}^{\infty}\left(n+\frac{1}{3}\right) e^{3 \pi i\left(n+\frac{1}{3}\right)^{2} \tau} .
\end{aligned}
$$


Theorem 4.1 (Zwegers [22]). Let $z \in \mathbb{H}$. The function $H(z):=F(z)-G(z)$ is a vector-valued real analytic modular form of weight $1 / 2$ satisfying

$$
\begin{aligned}
& H(z+1)=\left(\begin{array}{ccc}
e(-1 / 24) & 0 & 0 \\
0 & 0 & e(1 / 3) \\
0 & e(1 / 3) & 0
\end{array}\right) H(z), \\
& H(-1 / z)=\sqrt{-i z} \cdot\left(\begin{array}{ccc}
0 & 1 & 0 \\
1 & 0 & 0 \\
0 & 0 & -1
\end{array}\right) H(z) .
\end{aligned}
$$

Furthermore, $H(z)$ is an eigenfunction of the Casimir operator $\Omega_{\frac{1}{2}}$ with eigenvalue $\frac{3}{16}$, where $\Omega_{k}:=\Delta_{k}+\frac{2 k-k^{2}}{4}$.

In particular, if we write

$$
H(z)=\left(H_{0}(z), H_{1}(z), H_{2}(z)\right)^{T},
$$

then Theorem 4.1 implies the following result. Recall that $\chi_{12}(\bullet):=\left(\frac{12}{\bullet}\right)$.

Corollary 4.2. The function $H_{1}(6 z)$ is a good harmonic weak Maass form of weight $1 / 2$ on $\Gamma_{0}(144)$ with Nebentypus $\chi_{12}$.

Proof. Note first that by a change of variables,

$$
H_{1}(6 z)=2 q^{2} \omega\left(q^{3}\right)-2 \sqrt{2} i \int_{-\bar{z}}^{i \infty} \frac{\left(\theta_{6}(\tau)+2 \theta_{3} \mid V_{4}(\tau)\right) d \tau}{\sqrt{-i(\tau+z)}},
$$

where for $j \in\{3,6\}$,

$$
\chi_{j}(n):= \begin{cases}1 & \text { if } n \equiv 1 \quad(\bmod j) \\ -1 & \text { if } n \equiv-1 \quad(\bmod j)\end{cases}
$$

and

$$
\theta_{j}(z):=\sum_{n=0}^{\infty} \chi_{j}(n) n q^{n^{2}}
$$

We begin by verifying that $H_{1}(6 z)$ satisfies the proper transformation property. For $\left(\begin{array}{ll}a & b \\ c & d\end{array}\right) \in \Gamma_{0}(144)$, Theorem 4.1 implies that

$$
H_{1}\left(6 \frac{a z+b}{c z+d}\right)=H_{1}\left(\frac{a(6 z)+6 b}{\frac{c}{6}(6 z)+d}\right)=\epsilon(a, b, c, d)(c z+d)^{1 / 2} H_{i}(6 z)
$$

for some $i \in\{0,1,2\}$, where $\epsilon(a, b, c, d)^{24}=1$.

We now evaluate the period integral portion of $H_{1}(6 z)$ at $\frac{a z+b}{c z+d}$. Under the change of variables $\tau \mapsto \frac{a t-b}{-c t+d}$ this is

$$
\int_{-\bar{z}}^{d / c}\left(-i\left(\frac{a t-b}{-c t+d}+\frac{a z+b}{c z+d}\right)\right)^{-\frac{1}{2}}\left(\theta_{6}\left(\frac{a t-b}{-c t+d}\right)+2 \theta_{3} \mid V_{4}\left(\frac{a t-b}{-c t+d}\right)\right) \frac{d t}{(c t-d)^{2}} .
$$

Using the transformation properties of $\theta_{6}(\tau)+2 \theta_{3} \mid V_{4}(\tau) \in S_{3 / 2}\left(\Gamma_{0}(144), \chi_{12}\right)$ (see Chapters 1 and 3 of [17]) we can rewrite this integral as

$$
\chi_{12}(d)\left(\frac{-c}{d}\right)^{3} \epsilon_{d}^{-3}(c z+d)^{1 / 2} \int_{-\bar{z}}^{d / c} \frac{\left(\theta_{6}(t)+2 \theta_{3} \mid V_{4}(t)\right) d t}{\sqrt{-i(t+z)}} .
$$


Note that $\left(\frac{-c}{d}\right)^{3} \epsilon_{d}^{-3}=\left(\frac{c}{d}\right) \epsilon_{d}^{-1}$.

The corresponding transformation for $F_{1}(6 z)$ is given by Theorem 2.4 in [5]. Combining this with the transformation of $G_{1}(6 z)$ yields

$$
\begin{aligned}
H_{1}\left(6 \frac{a z+b}{c z+d}\right) & =i^{-3 / 2}(-1)^{(a+1) / 2} e\left(\frac{9 a b}{4}+\frac{17(a+d)}{4 c}\right) \omega_{-d, c / 6}^{-1}(c z+d)^{1 / 2} 2 q^{2} \omega\left(q^{3}\right) \\
- & \frac{12}{c}(c z+d) e\left(\frac{11 a}{4 c}\right) \sum_{\nu}(-1)^{\nu} \exp \left\{-\frac{18 \pi a(\nu+1 / 2)^{2}}{c}\right\} \\
& \times \int_{-\infty}^{\infty} \frac{\exp \left\{\frac{18 \pi i(c z+d) x^{2}}{c}\right\} d x}{\tanh \left(\frac{6 \pi i(v+1 / 3)}{c}+\frac{6 \pi i(c z+d) x}{c}\right)} \\
+ & 2 \sqrt{2} i \chi_{12}(d)\left(\frac{c}{d}\right) \epsilon_{d}^{-1}(c z+d)^{1 / 2} \int_{d / c}^{i \infty} \frac{\left(\theta_{6}(\tau)+2 \theta_{3} \mid V_{4}(\tau)\right) d \tau}{\sqrt{-i(\tau+z)}} \\
- & 2 \sqrt{2} i \chi_{12}(d)\left(\frac{c}{d}\right) \epsilon_{d}^{-1}(c z+d)^{1 / 2} \int_{-\bar{z}}^{i \infty} \frac{\left(\theta_{6}(\tau)+2 \theta_{3} \mid V_{4}(\tau)\right) d \tau}{\sqrt{-i(\tau+z)}}
\end{aligned}
$$

Here $\omega_{h, k}:=e^{\pi i s(h, k)}$, where $s(h, k)$ is the usual Dedekind sum. In particular, the last summand on the right hand side is the non-holomorphic contribution. By comparing this with $G_{j}(6 z)$ for the various $j$ we see that $i=1$ and $\epsilon(a, b, c, d)=\chi_{12}(d)\left(\frac{c}{d}\right) \epsilon_{d}^{-1}$.

Now that we know how $H_{1}(6 z)$ transforms, consider the other conditions in Definition 2.1. By Theorem 4.1 we have $\Delta_{1 / 2} H_{1}(6 z)=0$. Moreover, the transformation property given above along with the exact formula for the Fourier expansion at infinity of $H_{1}(z)$ given in [14] implies that $H_{1}(6 z)$ has at most linear exponential growth at the cusps. Thus, $H_{1}(6 z)$ is a harmonic weak Maass form of weight $1 / 2$ on $\Gamma_{0}(144)$ with Nebentypus $\chi_{12}$.

Now that we have $2 q^{2} \omega\left(q^{3}\right)$ as part of the good harmonic weak Maass form $H_{1}(6 z)$, we can apply Theorem 1.1 and Theorem 1.2 to obtain congruence and distribution properties for $\alpha_{\omega}(n)$. For example, we have the following immediate corollary of Theorem 1.1.

Corollary 4.3. For any prime $p \geq 5$, any prime $\ell \nmid 6 p$ and any $j \geq 1$, there exists a positive integer $m$ such that a positive proportion of the primes $Q$ have the property that

$$
\alpha_{\omega}\left(\frac{Q^{3} \ell^{m} n-2}{3}\right) \equiv 0 \quad\left(\bmod \ell^{j}\right)
$$

for all $n$ with $(n, \ell Q)=1$ and $\chi_{p}\left(-Q^{3} \ell^{m} n\right)=-1$.

Remark. The existence of the congruences (1.4) follows from Corollary 4.3, the Chinese Remainder Theorem and the fact that $Q \equiv-1\left(\bmod 144 N p^{4} \ell^{j+2}\right)$.

\section{Acknowledgements}

The authors would like to thank Ken Ono for his comments on this paper and for keeping us informed of developments in the theory of Maass forms. The authors also wish to thank Kathrin Bringmann and the referee for their insightful feedback. 


\section{References}

[1] S. Ahlgren, Distribution of the partition function modulo composite integers $M$, Math. Ann. 318 (2000), no. 4, 795-803.

[2] S. Ahlgren and M. Boylan, Arithmetic properties of the partition function, Invent. Math. 153 (2003), no. 3, 487-502.

[3] — Coefficients of half-integral weight modular forms modulo $\ell^{j}$, Math. Ann. 331 (2005), no. $1,219-239$

[4] S. Ahlgren and K. Ono, Congruence properties for the partition function, Proc. Natl. Acad. Sci. USA 98 (2001), no. 23, 12882-12884 (electronic).

[5] G. E. Andrews, On the theorems of Watson and Dragonette for Ramanujan's mock theta functions, Amer. J. Math. 88 (1966) 454-490.

[6] K. Bringmann, On certain congruences for Dyson's ranks. Accepted for publication, Int. J. Number Theory.

[7] K. Bringmann, J. Bruinier, A. Folsom, and K. Ono, Harmonic Maass forms and Borcherds products. Preprint.

[8] K. Bringmann, A. Folsom, and K. Ono, q-series and weight 3/2 Maass forms. Preprint.

[9] K. Bringmann and K. Ono, Dyson's ranks and Maass forms. Accepted for publication, Ann. of Math.

[10] - The $f(q)$ mock theta function conjecture and partition ranks, Invent. Math. 165 (2006), no. $2,243-266$.

[11] K. Bringmann, K. Ono, and R. C. Rhoades, Eulerian series as modular forms. Accepted for publication, J. Amer. Math. Soc.

[12] J. H. Bruinier and K. Ono, Coefficients of half-integral weight modular forms, J. Number Theory 99 (2003), no. 1, 164-179.

[13] D. Choi, Distribution of integral Fourier coefficients of a modular form of half integral weight modulo primes. ArXiv:0704.0012v1 [math.NT].

[14] S. A. Garthwaite, The coefficients of the $\omega(q)$ mock theta function. Accepted for publication, Int. J. Number Theory.

[15] K. Ono, Mock theta functions, ranks, and Maass forms. Accepted for publication in Proceedings of the special year in number theory and combinatorics (2004-2005) at the University of Florida, Springer.

[16] - Distribution of the partition function modulo m, Ann. of Math. (2) 151 (2000), no. 1, 293-307.

[17] - The web of modularity: arithmetic of the coefficients of modular forms and $q$-series, Vol. 102 of CBMS Regional Conference Series in Mathematics, Published for the Conference Board of the Mathematical Sciences, Washington, DC (2004), ISBN 0-8218-3368-5.

[18] S. Ramanujan, The lost notebook and other unpublished papers, Springer-Verlag, Berlin (1988), ISBN 3-540-18726-X. With an introduction by George E. Andrews.

[19] S. Treneer, Quadratic twists and the coefficients of weakly holomorphic modular forms. Preprint.

[20] - Congruences for the coefficients of weakly holomorphic modular forms, Proc. London Math. Soc. (3) 93 (2006), no. 2, 304-324.

[21] S. Zwegers, Mock Theta Functions, Ph.D. thesis, Universiteit Utrecht (2002).

[22] S. P. Zwegers, Mock $\theta$-functions and real analytic modular forms, in $q$-series with applications to combinatorics, number theory, and physics (Urbana, IL, 2000), Vol. 291 of Contemp. Math., 269-277, Amer. Math. Soc., Providence, RI (2001).

Department of Mathematics, Bucknell University, Lewisburg, PA 17870

E-mail address: sharon.garthwaite@bucknell.edu

Department of Mathematics, Furman University, Greenville, SC 29613

E-mail address: david.penniston@furman.edu 